CORRESPONDENCE

DOI: $10.1038 / \mathrm{s} 41467-018-07252-4$

\title{
Reply to 'Dissimilarity measures affected by richness differences yield biased delimitations of biogeographic realms'
}

Mark J. Costello (1) ${ }^{1,2}$, Peter Tsai (iD ${ }^{2}$, Alan Kwok Lun Cheung ${ }^{3}$, Zeenatul Basher ${ }^{1} \&$ Chhaya Chaudhary ${ }^{1}$

Recently, we classified the oceans into 30 biogeographic realms based on species' endemicity. Castro-Insua et al. criticize the choices of dissimilarity coefficients and clustering approaches used in our paper, and reanalyse the data using alternative techniques. Here, we explain how the approaches used in our original paper yield results in line with existing biogeographical knowledge and are robust to alternative methods of analysis. We also repeat the analysis using several similarity coefficients and clustering algorithms, and a neural network theory method. Although each combination of methods produces outputs differing in detail, the overall pattern of realms is similar. The coarse nature of the present boundaries of the realms reflects the limited field data but may be improved with additional data and mapping to environmental variables.

\footnotetext{
${ }^{1}$ Institute of Marine Science, University of Auckland, Auckland 1142, New Zealand. ${ }^{2}$ Bioinformatics Institute, University of Auckland, Auckland 1142, New Zealand. ${ }^{3}$ School of Environment, University of Auckland, Auckland 1142, New Zealand. Correspondence and requests for materials should be addressed to M.J.C. (email: m.costello@auckland.ac.nz)
} 
$\mathrm{R}$ ecognising the data limitations for the ocean ${ }^{1-3}$, an ideal biogeographic mapping would combine both empirical field data with expert knowledge of species ecology to account for sampling bias, and consider the validity of its findings to prior work, e.g., refs ${ }^{4-6}$. We used a world data set of 65,000 marine species mapped to $5^{\circ}$ latitude-longitude cells to map realms based on species endemicity ${ }^{1}$. Our primary presentation of the results used Jaccard's coefficient of similarity, by far the most widely used index in biogeography studies ${ }^{7}$, and Group Average linkage clustering methods. However, we also showed similar biogeographic patterns were obtained using equal area hexagons of different sizes, and alternative coefficients, including Simpson's coefficient that we noted is not biased by species richness (see Fig. 3 in ref. ${ }^{1}$ ). During preliminary analyses, we had explored numerous other coefficients and clustering methods, but either found them unsuitable or did not find biologically meaningful differences in the results. The three main steps in our analysis were statistical cluster analysis of a carefully curated data set, manual exclusion of geographically isolated cells surrounded by a more biogeographically coherent cluster, and comparison of the recommended realms to previously proposed classifications (see Supplementary Table 4 in ref. ${ }^{1}$ ). The data consisted of diverse marine species sampled by a wide variety of methods, from human observations in the field, to plankton nets, fishery trawls, and analysis of sediment samples. Thus variation in clusters could result from neighbouring cells appearing different because one had only benthic and another only pelagic samples for example, or distant cells appearing the same because their records were limited to widespread pelagic species.

Castro-Insua et al. ${ }^{2}$ re-analysed our data using several similarity coefficients, clustering algorithms and groupings of clusters. Their maps look like our map of the clusters before demarcation of the realms (see Supplementary Fig. 3 in ref. ${ }^{1}$ ). This map had over 200 potential clusters but we manually merged isolated clusters (e.g. only a single $5^{\circ}$ cell) into their neighbours because we did not consider them biogeographically robust and likely a reflection of sampling bias. We further examined the number of clusters at $1 \%$ similarity steps in the dendrogram until clusters showed no clear geographic relationship. This process was conservative and emphasised geographic coherency as a principle of the realms.

As suggested by Castro-Insua et al. ${ }^{2}$, we here compare how the use of Jaccard's and Simpson's coefficients, and Group Average and Ward's clustering methods, affect the hierarchical clustering of the realms. Indeed, differences in the cluster dendrograms do occur (Fig. 1). Jaccard's with Group Average singles out the Baltic Sea first from all other realms, and then the Black Sea (Fig. 1). Both 'seas' have low and variable salinity regions and thus freshwater species are present which distinguish them from all other realms. However, Jaccard's with Ward's coefficient places both seas' together and within a Pacific-Indian-Southern Ocean cluster which makes no biogeographic sense given the geographic distance between these locations. In contrast, Jaccard's with Group Average subsequently separates an Atlantic-Arctic (including part of the very North Pacific) group from a southern hemisphere group (including the Pacific, Southern and Indian Oceans). When we examine the dendrograms using Simpson's coefficient we find Ward's methods provide more biogeographically meaningful arrangements of the realms than using Group Average clustering (Fig. 1). In all cases with Simpson's, the Black Sea now clusters most closely with its Mediterranean neighbour, and the Baltic Sea with its neighbouring North-East Atlantic realm. This result also makes biogeographic sense, indicating how each coefficient differently emphasises species similarities and richness. In the case of Simpson's, we found that Ward's provides a more balanced hierarchy than Group Average, as suggested by ref. ${ }^{8}$, and it first separates the realms into two groups, a northern Atlantic-Pacific + Arctic, and mid and southern Pacific + Southern + Indian Ocean, clusters (Fig. 1).

A very different method for mapping endemicity, Infomaps, has also been developed recently using neural network theory ${ }^{8,9}$. In contrast to the above methods, it uses each species record (latitudelongitude) directly. We also used this method (see Fig. 3 in ref. ${ }^{1}$ ) and while similar to our proposed realms overall, it did extend the Caribbean realm southwards along the coast of Brazil. Here we map results of cluster analyses using both Sorenson's (similar to Jaccard's) and Simpson's coefficients with Group Average clustering, and Infomaps on both 2009 and a 2015 OBIS data set (used in $^{10}$ (Fig. 2). In contrast to our previous analysis, which included all $5^{\circ}$ cells with any data, when analysing the 2015 data, we excluded cells with fewer than 50 samples. These data exclusion explains the empty hexagon cells on the map in Fig. 2. While all maps show the same general distribution of realms, they also show how the realm boundaries need better resolution.

We find both Jaccard's and Sorenson's with Group Average clustering, and Simpson's with Ward's clustering, provide biogeographically informative outcomes, as does Infomaps. Cluster analysis is exploratory and results need to be interpreted based on understanding the underlying data to judge if clusters form meaningful groups. Our realms were defined by using both these statistical methods and knowledge of the species composition of the underlying data to consider sampling bias, and then compared with prior expert classifications (see Fig. 2 and Supplementary Table 4 in ref. ${ }^{1}$ ). The analytical alternatives suggested by Castro-Insua et al. are part of a suite of alternative methods of cluster analysis. The results of all of these methods need to be interpreted in the context of the taxonomic and geographic limitations of the underlying data, and in biogeography, in terms of geographic coherence and related knowledge.

The results of mapping the marine realms supported theories that pelagic, deep-sea and microscopic species are more widespread than benthic, coastal, and macroscopic, respectively ${ }^{11}$. Species' endemicity was higher along continental shelves than in the open ocean and deep-sea. These shelves provide most fisheries and suffer most human impact, and are a focus of conservation efforts. The map provides a practical resource for conservation and resource management, and a hypothesis, which should be tested with additional data and software tools. Indeed, independent data analyses support our classification for shallow-water ostracods ${ }^{12}$ and have split Australia into the same northern tropical and southern temperate realms as in our analysis ${ }^{13}$. We show and agree with Castro-Insua et al. that alternative statistical methods can affect the hierarchical relationships of the realms, and that biogeographic research needs to consider both the similarity coefficient, clustering algorithm, and number of clusters. However, we caution against using only one method because alternatives, including Jaccard's, Simpson's, Infomaps, combined with alternative clustering methods, may provide new insights into biogeographic patterns. Further, these patterns must somehow reflect environmental conditions, and such data are increasingly available in the four-dimensions of latitude, longitude, depth, and time $e^{14,15}$.

\section{Data availability}

The primary data used here are freely available from OBIS (https://www.iobis.org). The aggregated species by $5^{\circ}$ cell matrix finally used in the data analysis is available from Figshare at https://figshare.com/s/e11b3f7769ef353c6262 and https://doi.org/ 10.17608/k6.auckland.5086654

Received: 9 February 2018 Accepted: 19 October 2018 Published online: 30 November 2018 
a

Tropical Australia and Coral Sea Tropical Indo Pacific, coastal Indian Ocean Offshore W Pacific

Mid South Tropical Pacific Mid tropical North Pacific Ocean S Africa

Offshore Indian Ocea NW Pacific

Tasman Sea Zealand

S Australia

Red Sea

Offshore mid-E Pacific

SE Pacific

Tropical E Pacific
Chile

Southern Ocean

Argentina

Arctic Europe

N Atlantic boreal, sub-Arctic

North Pacific

Offshore and NW North Atlantic

NE Atlantic

Offshore S Atlantic

Tropical W Atlantic

Mediterranean

Tropical E Atlantic

Inner Baltic Se

C

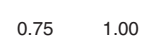

b

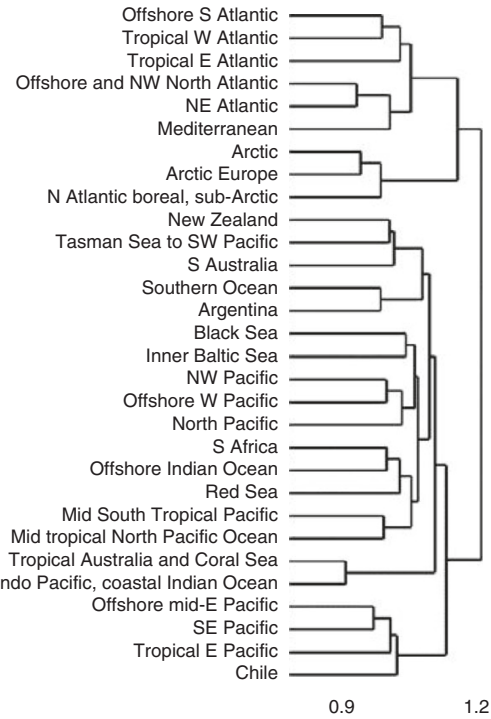

Tropical Indo Pacifc, costal Indian Oean

Red Sea

Offshore Indian Ocean

Mid South Tropical Pacific

S Africa
Mid tropical North Pacific Ocean
S Australia
Tropical Australia and Coral Sea

Tasman Sea to SW Pacific

NW Pacific

Offshore W Pacific

Offshore mid-E Pacific

Tropical E Pacific

North Pacific

North Pacific

NE Atlantic

Inner Baltic Sea

Arctic Europe
Tropical W Atlantic

Offshore and NW North Atlantic

$\mathrm{N}$ Atlantic boreal, sub-Arctic

Mediterranean

Black Sea

Tropical E Atlantic

Southern Ocean

Argentina

Chile

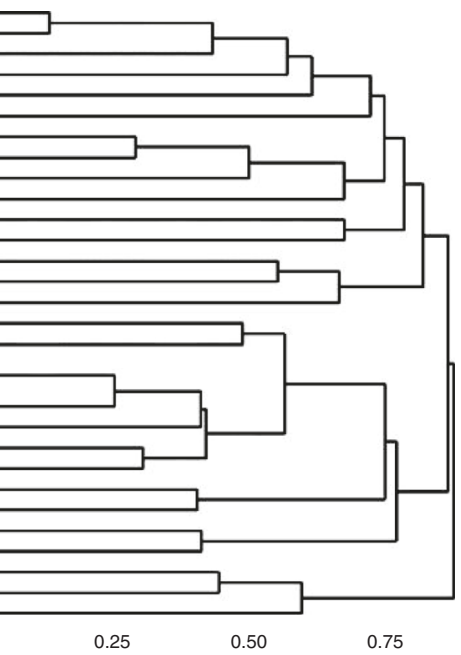

d

Red Sea
Tropical Indo Pacific, coastal Indian Ocean

$$
\begin{array}{r}
\text { Mid South Tropical Pacific } \\
\text { S Africa } \\
\text { NW Pacific }
\end{array}
$$

New Zealand

Tasman Sea to SW Pacific

$S$ Australia

S Australia
Tropical Australia and Coral Sea
Tropical E Pacific
Tropical W Atlantic

Offshore mid-E Pacific
Mid tropical North Pacific Ocean
Chile

Chile
SE Pacific

Tropical E Atlantic

Offshore S Atlantic

Southern Ocean

Argentina

NE Atlantic

Arctic Europe
Offshore and NW North Atlantic
N Atlantic boreal, sub-Arctic

N Atlantic boreal, sub-Arctic
North Pacific

North Pacific
Arctic
Mediterranean

Mediterranean
Black Sea

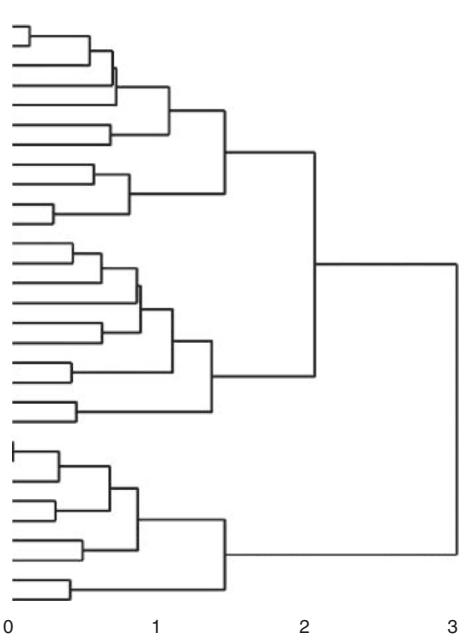

Fig. 1 Examples of analysis of hierarchical relationships between the realms using Jaccard's similarity coefficient with a Group Average and $\mathbf{b}$ Ward's clustering methods; and Simpson's similarity coefficient clustered using c Group Average and d Ward's clustering methods 

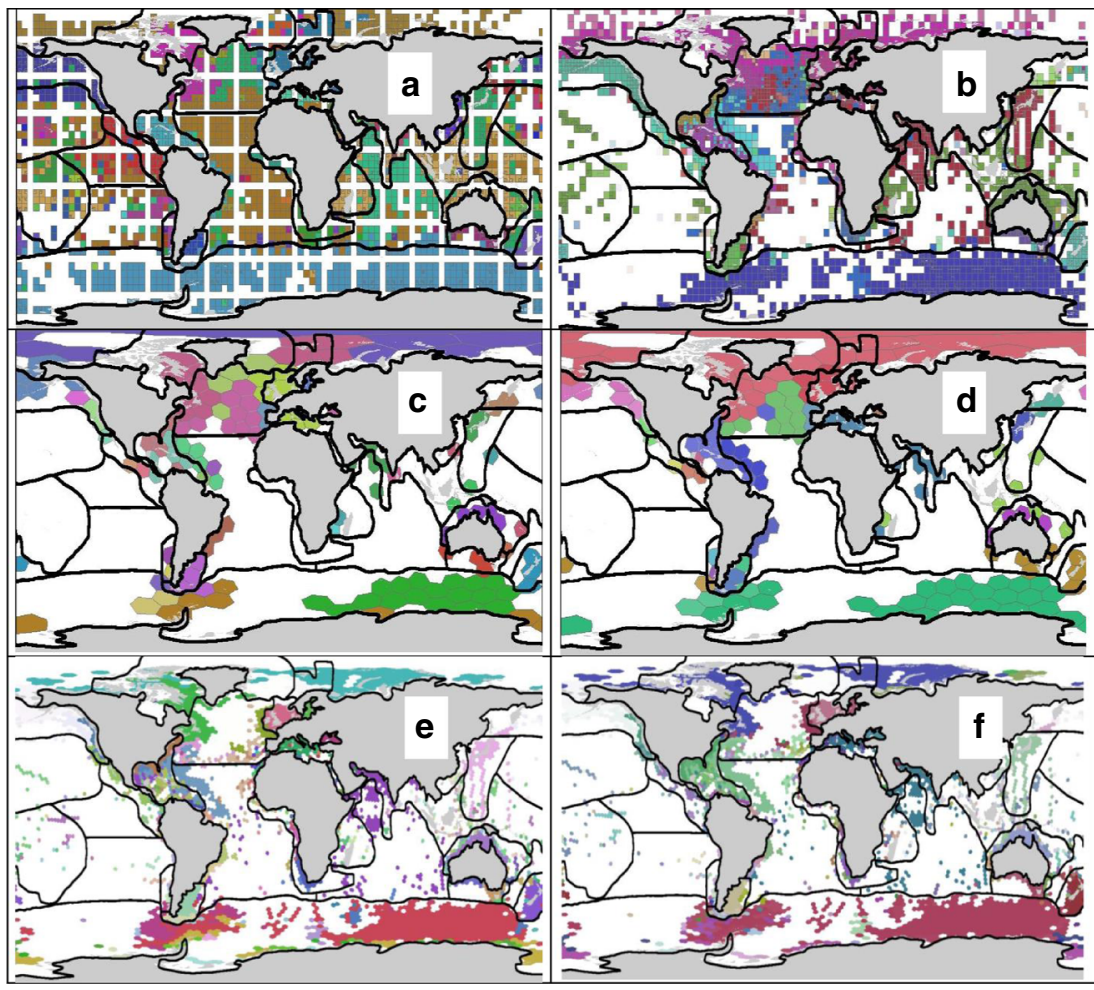

Fig. 2 Maps of the realms overlaid on results of biogeographic analyses. The biogeographic realms are denoted with black lines. Top row is using InfoMaps on the a 2009 ( $n=65,000$ species, 107 clusters) and b 2015 ( $n=51,670$ species, 140 clusters) data from OBIS. Results of analysis of the OBIS 2015 data is shown for two sizes of hexagons $\left(600,000-800,000 \mathrm{~km}^{2}\right.$ and $\sim 50,000 \mathrm{~km}^{2}$ ) using c Sorenson's (38 clusters, 0.8 similarity cutoff), d Simpson's ( 43 clusters, 0.8 cutoff), e Sorenson's ( 252 clusters, 0.86 cut off), and $\mathbf{f}$ Simpson's ( 252 clusters 0.75 cut off), similarity coefficients. The geographic gaps in b-f are because only cells with $>50$ samples (data with same time and place and one or more species) were used for analysis of the OBIS 2015 data. Colours were automatically assigned by the software to show cells belonging to the same biogeographic group, and are not comparable across maps

\section{References}

1. Costello, M. J. et al. Marine biogeographic realms and species endemicity. Nat. Commun. 8, 1057 (2017).

2. Castro-Insua, C. A., Gomez-Rodriguez, C., Baselga, A. Dissimilarity measures affected by richness differences yield biased delimitations of biogeographic realms. Nat. Commun. https://doi.org/10.1038/s41467-018-06291-1 (2018)

3. Costello, M. J., Vanhoorne, B. \& Appeltans, W. Conservation of biodiversity through taxonomy, data publication and collaborative infrastructures. Conserv. Biol. 29, 1094-1099 (2015).

4. Spalding, M. D. et al. Marine ecoregions of the world: a bioregionalization of coastal and shelf areas. BioScience 57, 573-583 (2007).

5. Ekman, S. Zoogeography of the Sea 417 (Sidgwick and Jackson, London, 1953).

6. Spalding, M. D., Agostini, V. N., Rice, J. \& Grant, S. M. Pelagic provinces of the world: a biogeographic classification of the world's surface pelagic waters. Ocean Coast. Manag. 60, 19-30 (2012).

7. Kreft, H. \& Jetz, W. A framework for delineating biogeographical regions based on species distributions. J. Biogeog. 37, 2029-2053 (2010).

8. Edler, D., Guedes, T., Zizka, A., Rosvall, M. \& Antonelli, A. Infomap Bioregions: Interactive mapping of biogeographical regions from species distributions. Syst. Biol. 66, 197-204 (2016).

9. Vilhena, D. A. \& Antonelli, A. A network approach for identifying and delimiting biogeographical regions. Nat. Comm. 6, 7848 (2015).

10. Chaudhary, C., Saeedi, H. \& Costello, M. J. Marine species richness is bimodal with latitude: a reply to Fernandez and Marques. Trends Ecol. Evol. 32, 234-237 (2017).

11. Costello, M. J. \& Chaudhary, C. Marine biodiversity, biogeography, deep-sea gradients, and conservation. Curr. Biol. 27, R511-R527 (2017).

12. Yasuhara, M. et al. Eocene shallow-marine ostracods from Madagascar: southern end of the Tethys? J. Syst. Palaeontol, 1-53, https://doi.org/10.1080/ 14772019.2018.1453555 (2018).

13. Cresswell, A. K. et al. Translating local benthic community structure to national biogenic reef habitat types. Glob. Ecol. Biogeogr. 26, 1112-1125 (2017).

14. Sayre, R. G. et al. A three-dimensional mapping of the ocean based on environmental data. Oceanography 30, 90-103 (2017).
15. Costello, M. J., Basher, Z., Sayre, R., Breyer, S. \& Wright, D. Stratifying ocean sampling globally and with depth to account for environmental variability. Sci. Rep. 8, 11259 (2018).

\section{Author contributions}

All present authors discussed the Response and designed this Reply. M.J.C. led writing and formatting of this paper. P.T. and C.C. conducted the present data analyses.

\section{Additional information}

Competing interests: The authors declare no competing interests.

Reprints and permission information is available online at http://npg.nature.com/ reprintsandpermissions/

Publisher's note: Springer Nature remains neutral with regard to jurisdictional claims in published maps and institutional affiliations.

\footnotetext{
Open Access This article is licensed under a Creative Commons Attribution 4.0 International License, which permits use, sharing adaptation, distribution and reproduction in any medium or format, as long as you give appropriate credit to the original author(s) and the source, provide a link to the Creative Commons license, and indicate if changes were made. The images or other third party material in this article are included in the article's Creative Commons license, unless indicated otherwise in a credit line to the material. If material is not included in the article's Creative Commons license and your intended use is not permitted by statutory regulation or exceeds the permitted use, you will need to obtain permission directly from the copyright holder. To view a copy of this license, visit http://creativecommons.org/ licenses/by/4.0/.
}

(c) The Author(s) 2018 